\title{
Visualization of Magnetic Structures in Superconductors and Magnetic Materials using Magneto-Optical Garnet Films
}

\author{
M.V. Indenbom ${ }^{1,2}$, L.S. Uspenskaya ${ }^{1}$, C.J. van der Beek ${ }^{2}$, and M. Konczykowski ${ }^{2}$ \\ ${ }^{1}$ Instititute for Solid State Physics RAS, 142432 Chernogolovka, Moscow distr., Russia \\ ${ }^{2}$ Laboratoire des Solides Irradiés, Ecole Polytechnique, CNRS UMR 7642 and CEA/DSM/DRECAM, 91128 Palaiseau, France
}

A short overview of the present state of the magneto-optical imaging of magnetic flux structures in superconductors and magnetic materials is given. Modern applications of the technique use ferrimagnetic garnet films with in-plane anisotropy as magneto-optical indicators. Advantages and limitations of the technique are reviewed and its possible further development is discussed.

Key words: magneto-optical imaging, magnetic materials, superconductors, thin films, magnetic structure, crystal defects, non-destructive control.

\section{Introduction}

In the last decade visualization of the magnetic flux structures using magneto-optical garnet films has become very popular for investigations of high $T_{c}$ superconductors. Many interesting results have been obtained both in fundamental studies of vortex matter ${ }^{1-8)}$ and in the exploration of new superconducting cables, bulk materials and thin film devices. ${ }^{8)-13)}$ The indicator films, initially designed for the non-destructive control of magnetic recordings, and rediscovered for the study of high $T_{c}$ superconductors, have recently been applied for magnetism once again: materials with colossal magnetoresistance and magnetostriction, ultra thin magnetic films and multilayers have been investigated. ${ }^{14)-17)}$ The progress in the application of magneto-optical imaging is rapid and continuous, and even a very recent extensive review involving only studies of superconductors ${ }^{8)}$ already cannot be considered as complete. Here we give some characteristic examples of recent results and discuss the limitations of the technique and its possible further development.

\section{Magneto-optical flux imaging technique}

The basic idea of magneto-optical flux imaging in media where direct magneto-optical imaging cannot be used (no magneto-optical effect, like in superconductors, or magnetic materials with bad surface or microgranular structure), has been known for many years. Magneto-optically inactive samples can bc covered by a magneto-optical material, whose magnetization serves as an indicator of the magnetic field distribution; this then can be visualized by a traditional magneto-optical scheme. But the magneto-optical materials found for intermetallic superconductors were active only at low temperatures and could not satisfy the new demands once the high- $\mathrm{T}_{\mathrm{c}}$ superconductors had been discovered. The application of garnet films has resolved the problem. ${ }^{1)}$ All recent results are obtained using garnet films with in-plane magnetic anisotropy, these films have become the major indicator material for magneto-optical imaging worldwide. ${ }^{3)-19)}$

For low temperature studies the sandwich, consisting of the sample and the indicator film on top of it, is placed in a special optical cryostat, with a small distance between the sample and the polarizing microscope objective. The usual parameters of the technique are the following: a spatial resolution of $1-5 \mu \mathrm{m}$, a field sensitivity of the order of $0.1 \mathrm{mT}$ and the maximum field of the order of $0.2 \mathrm{~T}$. Very recently a unique sensitivity and submicron resolution have been achieved, and the real-time dynamics of single Abrikosov vortices has been observed. ${ }^{7)}$

The cryogenic observations developed for superconductors are also useful for investigations of magnetic materials, even if their Curie temperature is well above the room temperature. At low temperatures the magnetic activation processes are slower and, thus, can be followed visually with higher precision.

The technique is not only for imaging, but also can be used for local quantitative magnetic measurements. The optical registration of the indicator magnetization in a spot, selected at a desired point of the observed picture, provides a local magnetometer, which can measure local magnetization curves or local AC response of the sample (see ${ }^{19)}$ and references there in). The analysis of the stray field distribution around small samples, occasional defects or specially etched circular holes in magnetic thin films provides both the value and the direction of the in-plane magnetization that is equivalent to a local vector magnetometer. ${ }^{15), 17), 19)}$

\section{High temperature superconductors}

Observation of magnetic flux dynamics in superconductors is a powerful tool for the verification of the nature of their electro-magnetic properties. For example, using a direct study of flux penetration into heavy-ion irradiated $\mathrm{YBa}_{2} \mathrm{Cu}_{3} \mathrm{O}_{7}$ crystals with controlled thickness variations, we have shown that the irradiation-induced amorphous columnar tracks create a gigantic surface pinning rather than the commonly supposed bulk pinning. ${ }^{6}$ ) A real picture of the topology of the vortex-lattice melting process in top quality $\mathrm{Bi}_{2} \mathrm{Sr}_{2} \mathrm{CaCu}_{2} \mathrm{O}_{8}$ crystals appeared to be unexpectedly inhomogeneous. ${ }^{4)}$ An unusual wavy motion of bundles of twisted vortices, which cannot be revealed by macroscopic measurements, was observed using magneto-optical imaging. ${ }^{3)}$ 


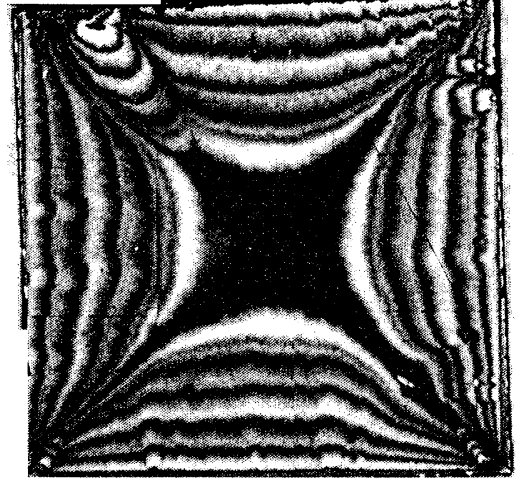

Fig.1. Image of successive flux fronts in a thin film of $\mathrm{YBa}_{2} \mathrm{Cu}_{3} \mathrm{O}_{7}$ superconductor after application of the magnetic field $+350 \mathrm{G}$, $-300 \mathrm{G},+250 \mathrm{G}, \ldots,+50 \mathrm{G}$. Size of the film is $10 \times 10 \mathrm{~mm}^{2}$; $\mathrm{T}=30 \mathrm{~K}$.

Observations of the magnetic flux structure in superconducting wires and melt-textured bulk ceramics give one the possibility to reconstruct the current paths in these new materials, and reveal the main obstacles for current flow, e.g. grain boundaries. ${ }^{9)-13)}$ Nowadays, it is difficult to imagine the development of applications of superconductors and the quality control of scientific samples without magneto-optical imaging. For demonstration, we compare here different thin films of the $\mathrm{YBa}_{2} \mathrm{Cu}_{3} \mathrm{O}_{7}$ high temperature superconductor.

In Fig. 1 a trapped flux structure in a $\mathrm{YBa}_{2} \mathrm{Cu}_{3} \mathrm{O}_{7}$ thin film square after the application of an alternating field with decreasing amplitude is shown. The general shape of the revealed flux contours was not correctly explained for more than 30 years, in spite of many similar observations using early realizations of magneto-optical imaging and decoration techniques. Now the shape of the flux fronts is well understood in terms of the strongly nonlinear electrodynamics and the flat shape of the sample. ${ }^{18)}$ Irregularities of the presented successive flux fronts reveal some defects in the sample, located mainly at the edges (E.g. the big defect at the left end of the upper edge). Interestingly, the distortion of the flux penetration emanates to a much bigger distance towards the sample center than the size of the defect. The rather regular flux structure in Fig. 1 proves that the investigated $\mathrm{YBa}_{2} \mathrm{Cu}_{3} \mathrm{O}_{7}$ film has a very high quality for its size.

Such a good film homogeneity is not always found. Many frequently appearing examples of unexpected irregularities of apparently crystallographically perfect $\mathrm{YBa}_{2} \mathrm{Cu}_{3} \mathrm{O}_{7}$ films (as checked, e.g. by X-ray diffraction) can be found in the recent review ${ }^{8}$ and references therein. Here we present a case which, to our knowledge, was never well documented in the literature. Sometimes we observe in $\mathrm{YBa}_{2} \mathrm{Cu}_{3} \mathrm{O}_{7}$ films a zigzag or parquet-like flux penetration (Fig. 2a). An ordinary inspection of the film under an optical microscope shows a shiny flat surface without defects, but under polarized light one can see narrow intergrowths (Fig. 2b). They may unexpectedly appear only in a part of the sample, while other part remains perfect. Using a polarized-optics analysis of the optical phase shift upon light reflection, we have determined that the crystallographic c-axis, which is oriented perpendicularly to the surface in the major part of the film, is oriented in the film plane and perpendicularly to the defect lines within the intergrowths. Clearly, the magnetic flux preferentially penetrates along the intergrowths. These defects can be easily missed without a special inspection; it is probably for this reason that there is little information about them.

One could imagine that perfect $\mathrm{YBa}_{2} \mathrm{Cu}_{3} \mathrm{O}_{7}$ single crystals, unlike thin films, demonstrate textbook flux dynamics, but it is not so. This high temperature superconductor has an orthorhombic crystal structure with a slight difference of the lattice parameters in the basal plane. From this follows a characteristic twin structure, which can be observed using reflected polarized light. Twin boundaries create an easy path for the motion of magnetic flux vortices along them and at the same time act as the barriers for the vortices crossing them. Magneto-optical imaging provides a unique possibility to see all details of this influence (see ${ }^{6}$ and references therein).

When additional strong defects are introduced in the superconductor by irradiation with heavy $\mathrm{Pb}$ ions, the influence of the twins is masked out and the flux penetration becomes homogeneous. ${ }^{6}$ ) This irradiation creates amorphous columnar tracks in the crystal. The columnar defects erase not only the influence of the twins, but also the effect of crystal thickness. The latter fact contradicts the Bean model for the flux penetration into type II superconductors, which predicts that the characteristic penetration field is proportional to the thickness. These particular observations are direct proof that the columnar defects create a gigantic surface pinning rather than an ordinary bulk pinning as is commonly believed. ${ }^{6}$

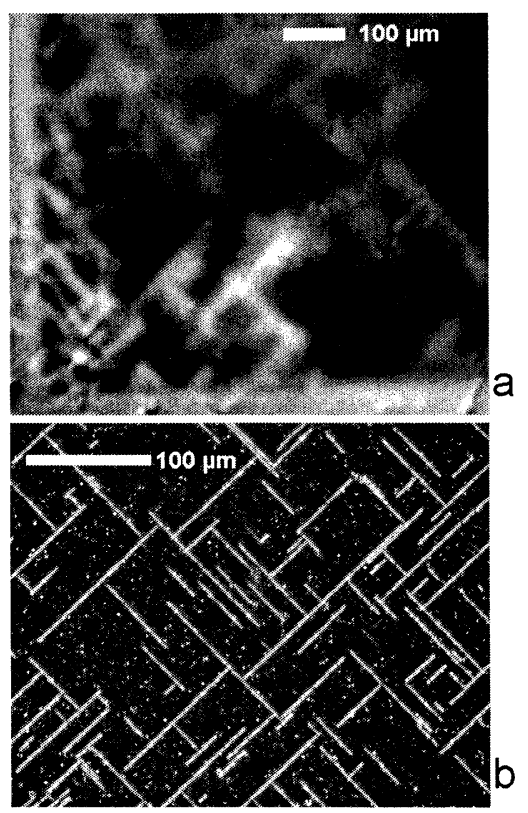

Fig.2. Influence of intergrowths onto flux penetration into an $\mathrm{YBa}_{2} \mathrm{Cu}_{3} \mathrm{O}$, thin film. a) Flux penetrated along intergrowths at $B=120 \mathrm{G}, \mathrm{T}=35 \mathrm{~K}$ (left bottom corner of the $1 \times 1 \mathrm{~mm}^{2}$ sample is shown). b) Intergrowths revealed on a part of the film surface in the reflected polarized light with a higher magnification. 
Magneto-optical imaging can compete well with other techniques of flux imaging in superconductors, due to its easier implementation. For example, scanning SQUID petrology using variations of $\mathrm{T}_{c}$ in an inhomogeneous material $^{20)}$ can be also realized with the magneto-optical technique. Our direct observations of the magnetic screening in $\mathrm{MgB}_{2}$, an old material in which superconductivity had just been discovered can be considered as realization of magneto-optical petrology. We have revealed a correlation between $\mathrm{Mg}$ deficiency and a small increase of $T_{c}$, which is very hard to find in a usual way. $^{19)}$

\section{Magnetic materials}

The "return" of magneto-optical imaging back to the investigation of magnetic materials has already provided a lot of interesting results which could form the topic of an extensive review. Like in the previous section, we will only give illustrations that outline the advantages of this technique. As for superconductors, imaging using magneto-optical garnet indicator films has appeared to be very useful both for the control of the real structure of new magnetic materials and for fundamental studies of magnetic exchange interactions in multilayers. In order to envisage the wide variety of the modern application of the magneto-optical imaging technique one can refer to observations of complicated dynamics of the domain structure in multilayers and thin films, ${ }^{14), 15)}$ the manifestation of a direct correlation of the magnetic structure with twins in $\mathrm{La}_{0.7} \mathrm{Sr}_{0.3} \mathrm{MnO}_{3}$ single crystals, ${ }^{16)}$ the demonstration of field-induced biquadratic exchange between soft $\mathrm{Fe}$ and hard SmCo thin layers using imaging of the local magnetization vectors. ${ }^{17)}$

As an illustration of sample structure control we present here an image of magnetic inhomogeneities induced in a $\mathrm{Fe} / \mathrm{Si} / \mathrm{Fe}$ multilayer thin film structure by twins in the substrate (see Fig. 3). The different lattice distortions of the different twin domains are inherited by the film and the corresponding stresses induce a variation of the magnetic anisotropy in the domains via the magnetostriction effect. We have to mention here that the distribution of the local magnetization of the sample is not necessarily identical to the observed distribution of the perpendicular magnetic field on the sample surface as frequently assumed. In this case it is very likely that the magnetization vectors remain in the film plane without any remarkable perpendicular component, and that the inhomogeneous perpendicular field appears due to stray fields at the boundaries of regions with different anisotropy and, thus, with different orientation of the magnetization. In order to obtain the distribution of the in-plane magnetization in the film from the observed perpendicular field distribution, one has to solve a reverse problem similar to that was solved in order to obtain the distributions of currents in superconductors from magneto-optical images (see recent review ${ }^{8)}$ and references therein). Note also, that the twin-induced magnetic patterns observed in Fig. 3 as well as in $\mathrm{La}_{0.7} \mathrm{Sr}_{0.3} \mathrm{MnO}_{3}$ single crystals ${ }^{16)}$ and other similar materials are not spontaneous magnetic domains.

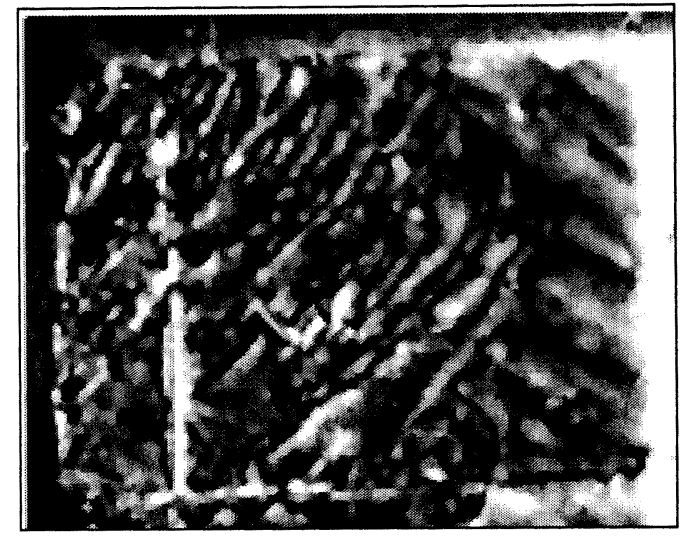

Fig.3. Magnetic structure due to twins in the substrate revealed in a $\mathrm{Fe} / \mathrm{Si} / \mathrm{Fe}$ multilayer film (50 layers, thickness $1 \mu \mathrm{m})$ in the in-plane magnetic field of $200 \mathrm{G}$ (applied horizontally in the figure).

When a magnetic sample is perfectly homogeneous and, seemingly, there is nothing to observe, the magneto-optical imaging can be very useful if one makes an artificial defect in the studied magnetic film. A circular hole prepared by photolithography, an example of which is shown in Fig. 4, can even be used for quantitative analysis. The stray field created by the homogeneous in-plane magnetization of the film at the edge of the hole is proportional to the magnetization projection onto a corresponding radius of the hole. The orientation of the symmetry axis of the characteristic picture presented in Fig. 4 shows the magnetization direction of the sample, and its intensity gives the magnetization value. This is equivalent to a vector magnetometer which provides direct information for studying the magnetic anisotropy and interlayer exchange interactions. Additionally one can directly see the appearance of the boundary of inhomogeneous remagnetization and interpret the data correctly at the moment, when the remagnetization boundary passes the measuring hole and the presented picture is distorted.

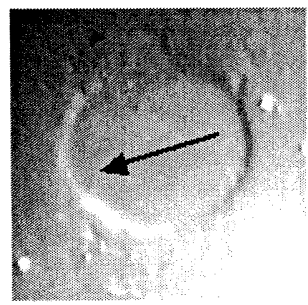

Fig. 4. Vector magnetometry using observations of the stray field around a circular hole in a $\mathrm{Fe} / \mathrm{Si} / \mathrm{Fe}$ multilayer film (10 layers, thickness $200 \mathrm{~nm}$ ). The in-plane field of $180 \mathrm{G}$ is applied in the horizontal direction. The magnetization direction shown by an arrow is revealed as the symmetry axis of the field distribution at the border of the hole, it clearly deviates from the applied field. The hole size is $200 \mu \mathrm{m}$. 


\section{Conclusions}

At present the applications of magneto-optical imaging using ferrimagnetic garnet films have already demonstrated a high effectiveness of this technique for both fundamental and applied studies of superconductors and magnetic materials. In particular we have to outline the relative simplicity of use of this imaging technique. It provides also a powerful tool for local quantitative magnetic measurements. For a correct interpretation of such measurements in the cases of inhomogeneous samples, one has to take into account the whole field distribution. The latter approach is already well developed for superconductors and should now be extended to magnetic materials.

Magneto-optical imaging using garnet indicators has some limitations which, nevertheless, allow it to be very competitive with other imaging techniques. The working field of the imaging is presently limited by about $0.2 \mathrm{~T}$. The development of indicators, that can be applied at higher fields but that will keep a comparable high sensitivity, is desirable.

We would like to acknowledge the people who provided us with samples used for the present demonstration: D.G. Crété, S. Berger, and J.P. Contour (Unité Mixte de Physique CNRS-Thales, Orsay, France) and P. Gierlowski (Institute of Physics PAS, Poland) for $\mathrm{YBa}_{2} \mathrm{Cu}_{3} \mathrm{O}_{7}$ films (Figs 1 and 2, correspondingly) and V.N. Matveev (Institute for Problems of Microelectronic Technology and High Purity Metals RAS, Chernogolovka, Russia) for Fe/Si/Fe multilayer films (Figs 3 and 4).

\section{References}

1) A.A.Polyanskii, V.K.Vlasko-Vlasov, M.V.Indenbom, and V.I. Nikitenko: Sov.Tech.Phys.Lett., 15, 872 (1989).

2) C.A. Durán, P.L. Gammel, R. Wolfe et al.: Nature, 357, 474 (1992).

3) M.V. Indenbom, C.J. van der Beek, V. Berseth et al.: Nature, 385, 702 (1997).

4) A. Soibel, E. Zeldov, M. Rappaport et al.: Nature 406, 282 (2000).

5) D. C. Larbalestier, L. D. Cooley, M. O. Rikel et al.: Nature, 410, 186 (2001)

6) M.V. Indenbom, C.J. van der Beek, M. Konczykowski et al.: Phys. Rev. Lett., 84, 1792 (2000).

7) P.E. Goa, H. Hauglin, M. Baziljevich et al.: Supercond. Sci. Technol., 14, 729 (2001).

8) Ch. Jooss et al.: Rep. Prog. Phys., 65, 651 (2002).

9) U. Welp, D.O. Gunter, G.W. Grabtree et al.: Nature, 376, 44 (1995).

10) X. Y. Cai, A. Polyanskii,Q.Li et al. : Nature 392, 906 (1988).

11) A.E. Pashitski, A.Gurevich, A.A. Polyanskii et al.: Science, 275, 367 (1997).

12) L.S. Uspenskaya, V.K. Vlasko-Vlasov, V.I. Nikitenko et al.: Phys.Rev.B, 56, 11979 (1997)

13) D. Larbalestier, A.Gurevich, D.W. Feldmann, and A. Polyanskii: Nature, 414, 368 (2001).

14) L.H. Bennett, R.D. McMichael, L.J. Swartzendruber et al.: Appl. Phys. Lett., 66, 888 (1995).

15) A. Khapikov, L. Uspenskaya, J. Ebothe and S. Vilain: Phys. Rev. B, 57, 14990 (1998).

16) A. Khapikov, L. Uspenskaya, I. Bdikin et al.: Appl. Phys. Lett. , 77, 2376 (2000).

17) V.K. Vlasko-Vlasov, U. Welp, J.S. Jiang et al.: Phys. Rev. Lett., 86, 4386 (2001).

18) Th. Schuster, H. Kuhn, E.H. Brandt et al.: Phys. Rev. B, 52, 10375 (1995).

19) M.V. Indenbom, L.S. Uspenskaya, M.P. Kulakov et al.: JETP Lett., 74, 274 (2001).

20) Bruce A. Scott, John R. Kirtley, David Walker et al: Nature, 389, 164 (1997).

Received June 6, 2002; Accepted August 22, 2002. 